\title{
ARTICLE
}

\section{Self-shielding effect of unresolved resonance data in JENDL-4.0}

\author{
Chikara Konno $^{\mathrm{a}^{*}}$, Yoshinari Kato ${ }^{\mathrm{b}}$, Kosuke Takakura ${ }^{\mathrm{a}}$, Masayuki Ohta ${ }^{\mathrm{a}}$, Kentaro Ochiai ${ }^{\mathrm{a}}$ and Satoshi Sato ${ }^{\mathrm{a}}$ \\ ${ }^{a} J a p a n$ Atomic Energy Agency, 2-4 Shirakata, Tokai-mura, Naka-gun, Ibaraki-ken, 319-1195, Japan; ${ }^{b}$ Tokai University, 4-1-1 \\ Kitakaname, Hiratsuka-shi, Kanagawa-ken, 259-1292, Japan
}

\begin{abstract}
In 2007 we pointed out that most of the unresolved resonance data in JENDL-3.3 had a problem related to self-shielding effect. Here through comparison of calculated neutron spectra from simple sphere assemblies and the TOF experiment for a manganese sphere at OKTAVIAN we investigate whether the latest JENDL, JENDL-4.0, is improved for the problem or not. The results for the spherical assemblies indicate that the unresolved resonance data in JENDL-4.0 are improved, but the data of the manganese TOF experiment at OKTAVIAN suggest that the unresolved resonance data in JENDL-4.0 still have some problems from the view of the self-shielding effect. New benchmark experiments such as deep penetration experiments are strongly recommended in order to validate the unresolved resonance data in JENDL-4.0.
\end{abstract}

Keywords: unresolved resonance data; self-shielding; JENDL-3.3; JENDL-4.0; MCNP

\section{Introduction}

In 2001 we calculated the spectra of neutrons which leaked from a niobium sphere of $0.5 \mathrm{~m}$ in radius with 20 $\mathrm{MeV}$ neutrons in the center by using the transport codes ANISN [1] and MCNP4C [2] and the nuclear data library JENDL-3.3 [3]. The calculated leakage neutron spectra had a strange bump at $100 \mathrm{keV}$ as shown in Figure 1 only when the unresolved resonance data were adopted [4]. It was concluded that the unresolved resonance data had some problems.

In 2007 we compared self-shielding corrected multigroup cross section data with and without the unresolved resonance data of ${ }^{93} \mathrm{Nb}$ in JENDL-3.3 [5]. Figure 2 shows them for the elastic scattering. It was found out that the self-shielding corrected elastic scattering cross section data had unphysical discontinuity around the maximum energy (100 keV) of the unresolved resonance region, which caused the strange bump at $100 \mathrm{keV}$ in Figure 1 [5]. We also checked other data in JENDL-3.3 and all data in other nuclear data libraries ENDF/B-VII.0 [6] and JEFF-3.1 [7]. It was pointed out that there are similar problems in unresolved resonance data in JENDL-3.3, ENDF/B-VII.0 and JEFF-3.1 [5].

JENDL-4.0 [8], the latest JENDL, tried to improve this problem by increasing the maximum energy of the unresolved resonance region. Note that unresolved resonance data are given to more nuclei in JENDL-4.0 (291 nuclei) than in JENDL-3.3 (210 nuclei), e.g. ${ }^{55} \mathrm{Mn}$. In this paper we examine whether the problem on the unresolved resonance data is really improved in

*Corresponding author. Email: konno.chikara@jaea.go.jp
JENDL-4.0 through comparison of calculated neutron

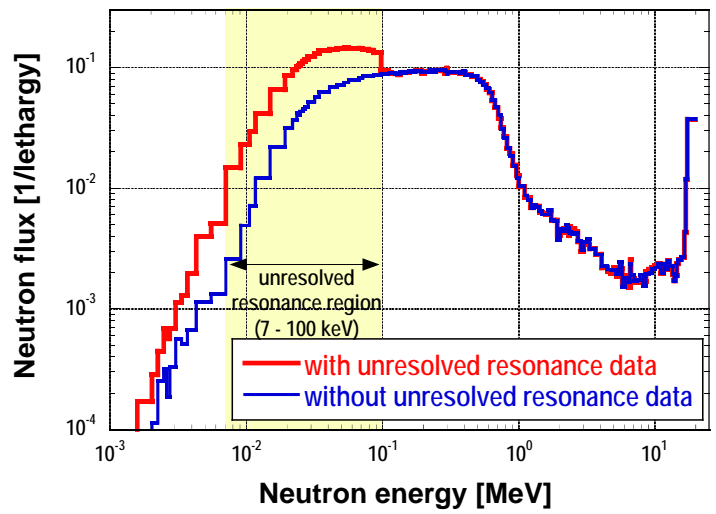

Figure 1. Calculated leakage neutron spectra from niobium sphere with MCNP and JENDL-3.3.

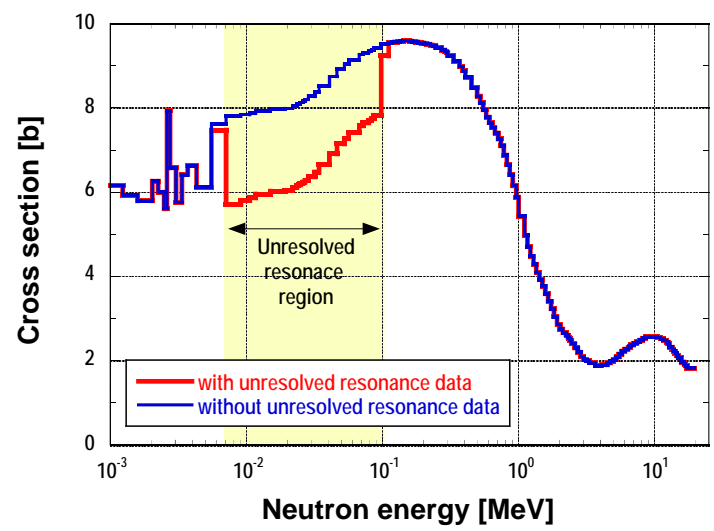

Figure 2. Elastic scattering cross section data of ${ }^{93} \mathrm{Nb}$ in JENDL-3.3. 
spectra from spherical assemblies and a benchmark experiment.

\section{Method}

We adopted the same sphere assembly as in the previous calculations, i.e. a natural element sphere of 0.5 $\mathrm{m}$ in radius with a $20 \mathrm{MeV}$ neutron source in the center. Pure manganese, arsenic and niobium were selected as the natural element because the self-shielding effect was expected to be enhanced owing to one stable isotope. Leakage neutron spectra from the sphere were calculated with the Monte Carlo code MCNP4C and the official ACE file FSXLIB-J40 [9] generated from JENDL-4.0 with the NJOY99.336 [10] code. We performed both analyses with and without the unresolved resonance data. Analyses with the official ACE file FSXLIB-J33 [11] generated from JENDL-3.3 with NJOY99.90 were also carried out for comparison.

The self-shielding effect for unresolved resonance data was examined with two multigroup libraries. One was produced from the official MATXS file MATXSLIB-J40 [9] with unresolved resonance data and the other was generated from a MATXS file without unresolved resonance data, which was produced from JENDL-4.0 by using NJOY99.336 with JENDL-4 patch. The self-shielding corrected elastic scattering cross section data with and without the unresolved resonance

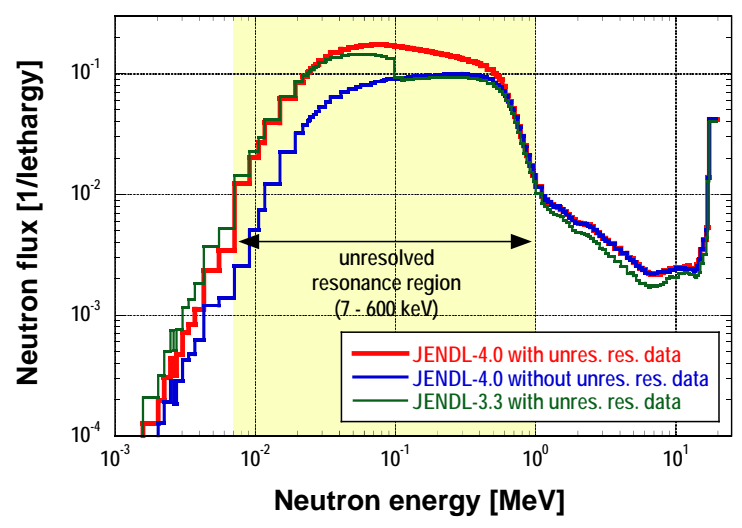

Figure 3. Leakage neutron spectra from niobium sphere.

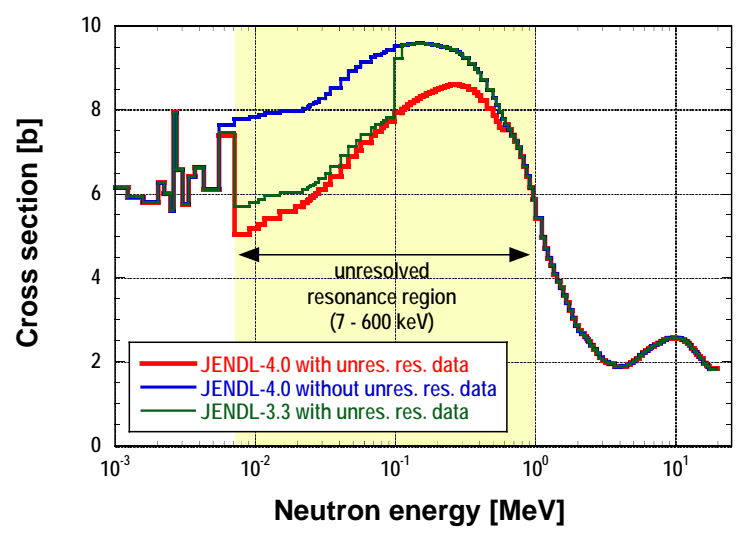

Figure 4. Elastic scattering cross section data of ${ }^{93} \mathrm{Nb}$. data were deduced from the two MATXS files with the TRANSX-2.15 [12] code. The self-shielding corrected one for JENDL-3.3 with the unresolved resonance data was deduced from the official MATXS file MATXSLIB-J33 [10].

In order to validate the unresolved resonance data in JENDL-4.0 we also analyzed the manganese sphere Time-Of-Flight (TOF) experiment at Osaka University/OKTAVIAN [13], which measured a spectrum of neutrons above $0.1 \mathrm{MeV}$ emitted from a manganese sphere of $61 \mathrm{~cm}$ in diameter bombarded with $14 \mathrm{MeV}$ neutrons.

\section{Results and discussion}

\subsection{Calculation with simple assembly of sphere}

\subsubsection{Niobium}

Figure 3 shows calculated leakage neutron spectra from the natural niobium sphere. The unresolved resonance region of ${ }^{93} \mathrm{Nb}$ is $7 \mathrm{keV}-600 \mathrm{keV}$ in JENDL-4.0, while it is $7 \mathrm{keV}-100 \mathrm{keV}$ in JENDL-3.3. The strange bump, which appears in the neutron spectrum with JENDL-3.3 around the maximum energy of the unresolved resonance region, disappears in that with JENDL-4.0 even with the unresolved resonance data. Figure 4 shows the self-shielding corrected elastic scattering cross section data of ${ }^{93} \mathrm{Nb}$ in both files with and without the unresolved resonance data. The self-shielding effect for the unresolved resonance data is larger in JENDL-4.0 than in JENDL-3.3, while we cannot judge whether the unresolved resonance data in JENDL-4.0 is improved or not from this result alone.

\subsubsection{Arsenic}

Figure 5 shows calculated leakage neutron spectra from the natural arsenic sphere. The unresolved resonance region of ${ }^{75} \mathrm{As}$ is $9.7 \mathrm{keV}-500 \mathrm{keV}$ in JENDL-4.0, while it is $9.7 \mathrm{keV}-100 \mathrm{keV}$ in JENDL-3.3. The strange bump, which appears in the neutron spectrum with JENDL-3.3 around the maximum energy of the unresolved resonance region, is very small in the spectrum employing JENDL-4.0 with the unresolved resonance data. Figure 6 shows the self-shielding

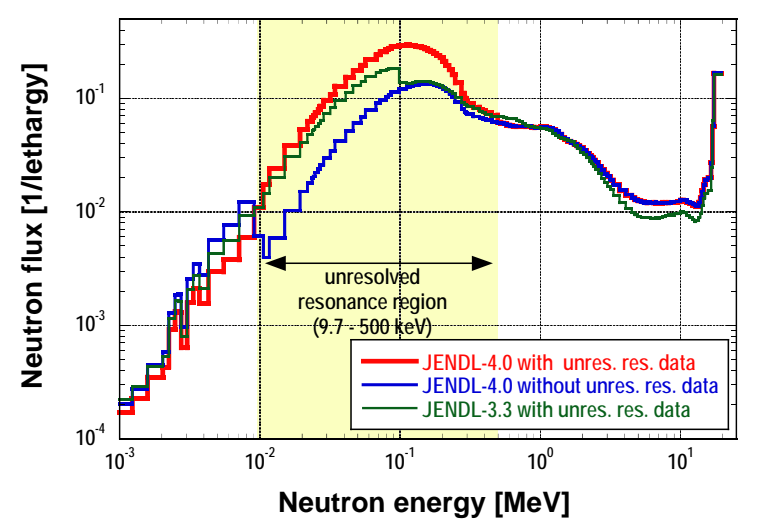

Figure 5. Leakage neutron spectra from arsenic sphere. 
corrected elastic scattering cross section data of ${ }^{75}$ As with and without the unresolved resonance data. As well as ${ }^{93} \mathrm{Nb}$, the self-shielding effect for the unresolved resonance data in JENDL-4.0 is very large.

\subsubsection{Manganese}

Figure 7 shows leakage neutron spectra from the natural manganese sphere. The unresolved resonance region of ${ }^{55} \mathrm{Mn}$ is $125 \mathrm{keV}-1 \mathrm{MeV}$ in JENDL-4.0, while ${ }^{55} \mathrm{Mn}$ in JENDL-3.3 has no unresolved resonance data. The spectra calculated using JENDL-4.0 without the unresolved resonance data are almost the same above

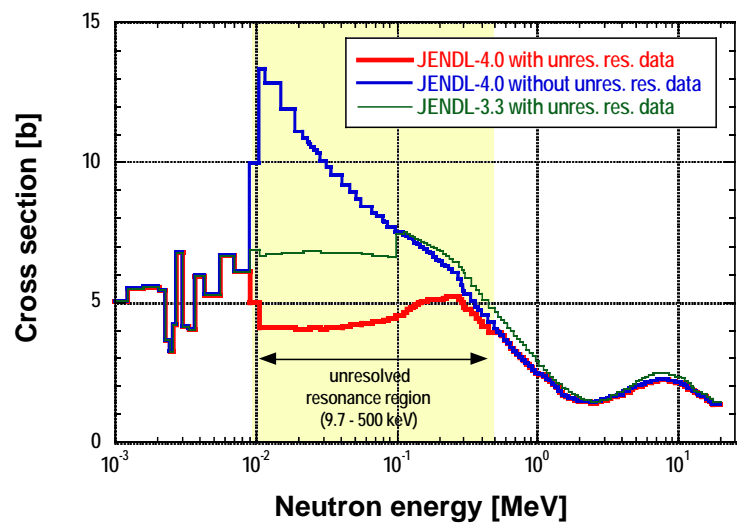

Figure 6. Elastic scattering cross section data of ${ }^{75}$ As.

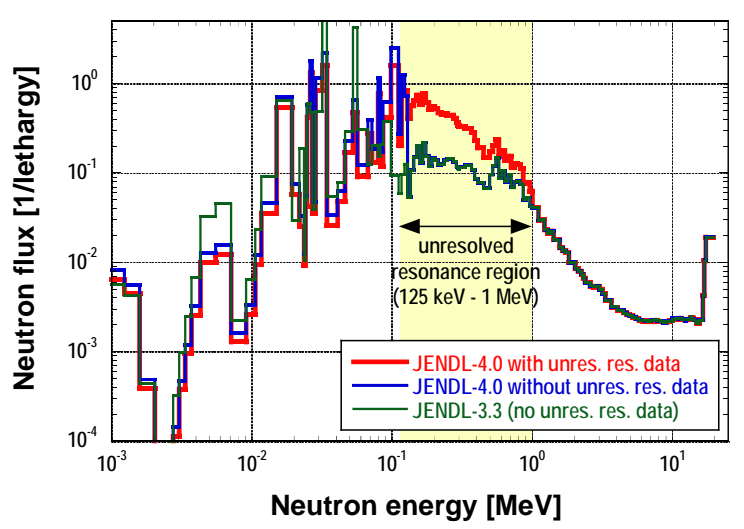

Figure 7. Leakage neutron spectra from manganese sphere.

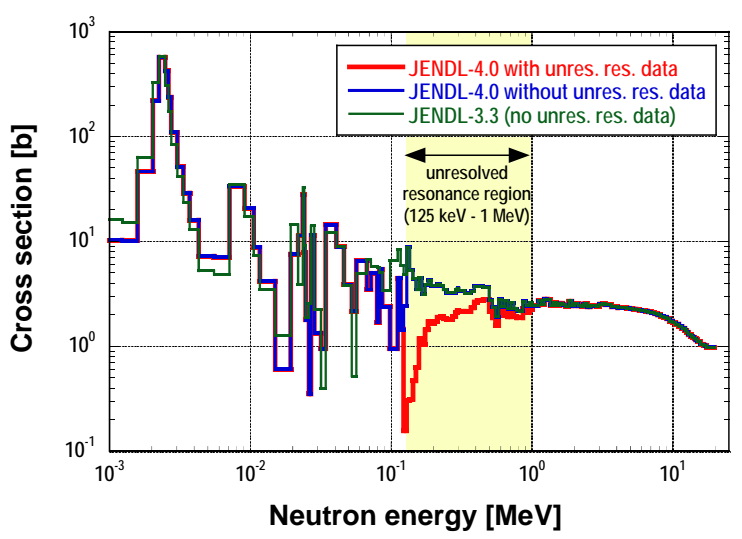

Figure 8. Elastic scattering cross section data of ${ }^{55} \mathrm{Mn}$.
$125 \mathrm{keV}$ as that using JENDL-3.3. There is no strange bump around the maximum energy of the unresolved resonance region in the neutron spectrum using JENDL-4.0 with unresolved resonance data. Figure 8 shows the self-shielding corrected elastic scattering cross section data of ${ }^{55} \mathrm{Mn}$ with and without the unresolved resonance data. As well as ${ }^{93} \mathrm{Nb}$ and ${ }^{75} \mathrm{As}$, the self-shielding effect in JENDL-4.0 is very large.

\subsection{Manganese TOF experiment at OKTAVIAN}

In order to judge whether the unresolved resonance data of manganese in JENDL-4.0 is better than no unresolved resonance data in JENDL-3.3, we analyzed the manganese sphere TOF experiment at OKTAVIAN. Figure 9 plots the measured and calculated leakage neutron spectra from the manganese sphere and the ratios of the calculated neutron fluxes to the measured ones for specified neutron energy regions. All the calculated neutron spectra above $1 \mathrm{MeV}$ are almost the same. On the other hand, below $1 \mathrm{MeV}$, the calculated neutron spectrum with JENDL-4.0 including the unresolved resonance data overestimates the measured one more than that with JENDL-4.0 excluding the unresolved resonance data. It seems that the unresolved resonance data are not necessary for ${ }^{55} \mathrm{Mn}$, as long as the data in Figure 9 are concerned.

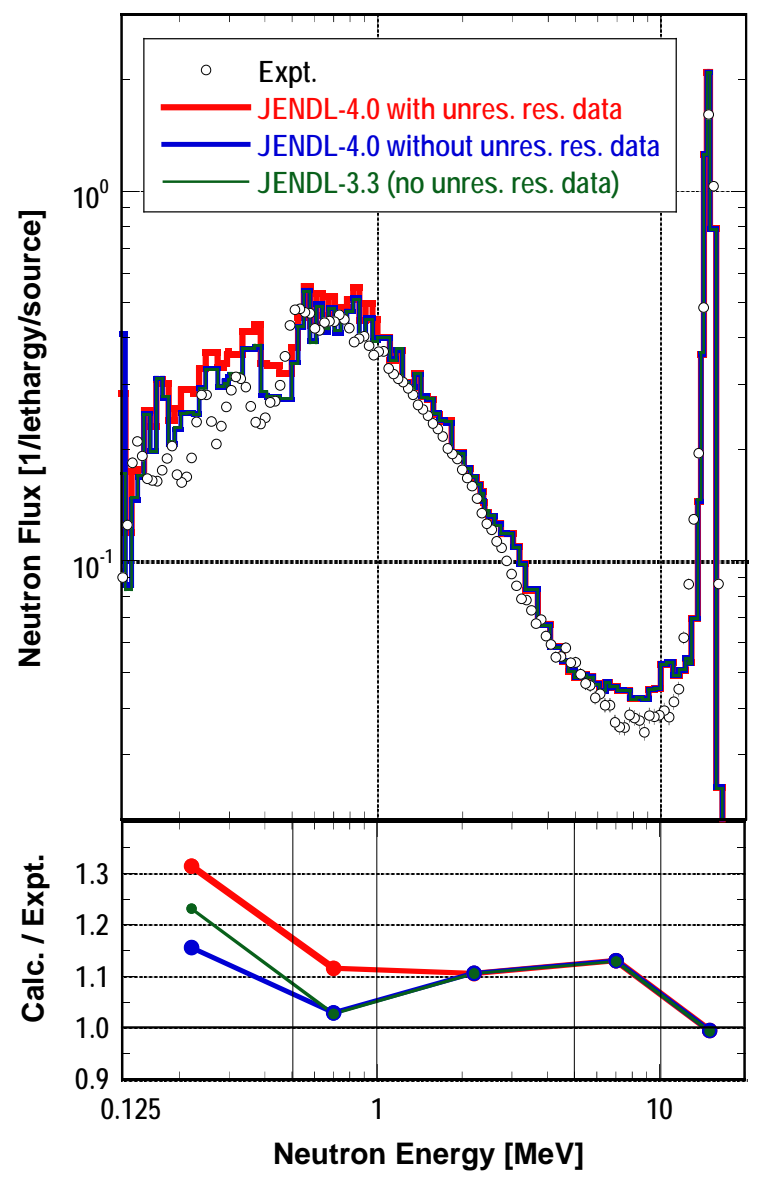

Figure 9. Leakage neutron spectra in manganese TOF experiment at OKTAVIAN. 


\section{Conclusion}

In order to investigate self-shielding effects of unresolved resonance data in JENDL-4.0, we have calculated leakage neutron spectra from natural manganese, arsenic and niobium sphere assemblies and have analyzed the manganese TOF experiment at OKTAVIAN. The results with the sphere assemblies indicate that the unresolved resonance data in JENDL-4.0 are improved, but the analysis of the manganese TOF experiment at OKTAVIAN suggests that the unresolved resonance data in JENDL-4.0 still have some problems from the view of the self-shielding effect. New benchmark experiments for elements with unresolved resonance data such as deep penetration experiments are strongly recommended in order to validate the unresolved resonance data.

\section{References}

[1] Oak Ridge National Laboratory, DOORS3.2 : One, Two- and Three-Dimensional Discrete Ordinates Neutron/Photon Transport Code System, RSICC CODE PACKAGE CCC-650, (1998).

[2] J.F. Briesmeister (Ed.), MCNP - A General Monte Carlo N-Particle Transport Code, Version 4C, LA-13709-M, Los Alamos National Laboratory, (2000).

[3] K. Shibata, T. Kawano, T. Nakagawa, et al., Japanese Evaluated Nuclear Data Library Version 3 Revision-3: JENDL-3.3, J. Nucl. Sci. Technol. 39 (2002), pp. 1125-1136.

[4] C. Konno and Y. Ikeda, MATXS files processed from JENDL-3.2 and -3.3 for shielding, J. Nucl. Sci. Technol. Suppl. 2 (2002), pp. 1037-1041.
[5] C. Konno, T. Nakagawa, K. Ochiai, S. Sato and M. Yamauchi, Problem on unresolved resonance data in recent nuclear data libraries, Proc. International Conference on Nuclear Data for Science and Technology 2007, April 22-27, 2007, Nice, France, (2008), pp.713-716, ISBN 978-2-7598-0090-2.

[6] M.B. Chadwick, P. Oblozinsky, M. Herman, et al., ENDF/B-VII.0: Next generation evaluated nuclear data library for nuclear science and technology, Nucl. Data Sheets 107 (2006), pp. 2931-3059.

[7] A. Koning, R. Forrest, M. Kellett, R. Mills, H. Henriksson and Y. Rugama (Eds.), The JEFF-3.1 Nuclear Data Library, JEFF Report 21, OECD NEA, (2006).

[8] K. Shibata, O. Iwamoto, T. Nakagawa, N. Iwamoto, A. Ichihara, S. Kunieda, S. Chiba, K. Furutaka, N. Otuka, T. Ohsawa, T. Murata, H. Matsunobu, A. Zukeran, S. Kamada and J. Katakura, JENDL-4.0: A new library for nuclear science and engineering, J. Nucl. Sci. Technol. 48 (2010), pp. 1-30.

[9] K. Okumura, private communication (2010).

[10]R.E. MacFarlane and D.W. Muir, The NJOY Nuclear Data Processing System, Version 91, LA-12740-M, Los Alamos National Laboratory, (1994).

[11]K. Kosako, N. Yamano, T. Fukahori, K. Shibata and A. Hasegawa, The Libraries FSXLIB and MATXSLIB based on JENDL-3.3, JAERI-Data/Code 2003-011, Japan Atomic Energy Research Institute, (2003).

[12]F. Maekawa, J. Yamamoto, C. Ichihara, K. Ueki and Y. Ikeda, Collection of Experimental Data for Fusion Neutronics Benchmark, JAERI-M 94-014, Japan Atomic Energy Research Institute, (1994). 